\title{
Scattering of a plane acoustic wave from a transversely isotropic cylinder encased in a solid elastic medium
}

\author{
Y. Fan and A. N. Sinclair ${ }^{\text {a) }}$ \\ Department of Mechanical and Industrial Engineering, University of Toronto, 5 Kings College Road, \\ Toronto, Ontario M5S 3G8, Canada \\ F. Honarvar \\ Department of Mechanical Engineering, K. N. Toosi University of Technology, P.O. Box 16765-3881, \\ Tehran 16579, Iran
}

(Received 3 April 1999; accepted for publication 28 May 1999)

\begin{abstract}
A mathematical model is developed to describe the scattering of a plane wave incident at an arbitrary angle on a transversely isotropic cylinder embedded in a solid elastic matrix. The model is based on the normal-mode expansion method, but is complicated by the coupling between the potential functions representing compressional and axially polarized shear waves. The solid matrix around the cylinder precludes the appearance of the leaky Rayleigh modes that dominate the spectrum of a cylinder immersed in a fluid. Instead, interfacial modes contribute to the scattered spectrum - these modes could be instrumental in the development of a nondestructive evaluation technique for the matrix-to-fiber bonds in a fiber-reinforced composite material. The presence of a solid matrix that supports shearing action leads to scattered compressional waves, and shear waves with polarization components in both the axial direction and $r-\theta$ planes. The sensitivity of resonances in each of these scattered wave components to perturbations in the cylinder's elastic constants is explored; results indicate that a judicious selection of resonance modes allows characterization of the cylinder's elastic properties. (C) 1999 Acoustical Society of America.
\end{abstract}

[S0001-4966(99)01609-4]

PACS numbers: 43.20.Bi, 43.20.Fn, 43.20.Ks [AN]

\section{INTRODUCTION}

The ultrasonic nondestructive evaluation (NDE) of fiber-reinforced composite materials is hampered by the highly complicated response function expected from even an undamaged specimen. The fibers are typically cylindrical in shape, each containing up to several transversely isotropic layers. The interface between the fiber and the surrounding elastic matrix must be strong, as a low pull-out strength will compromise the material's integrity under stress. In this paper, we determine the diffraction spectrum resulting from oblique incidence of a plane compression or shear wave on a single homogeneous cylinder with transversely isotropic symmetry, encased in a solid elastic matrix.

This effort is built upon a wealth of earlier work dealing with compression wave scattering from homogeneous, isotropic cylinders and shells immersed in a fluid. Following the pioneering work in this field by Faran ${ }^{1}$ and White, ${ }^{2}$ further studies by Flax and Neubauer, ${ }^{3}$ Nagl et al., ${ }^{4}$ and Veksler ${ }^{5}$ led to a broader understanding of the phenomenon. This included the development of Resonance Scattering Theory (RST) that explained the resonances in the scattered wave spectrum as the product of constructive interference among surface waves encircling the cylinder. Identification of the various wave modes, and their associated shapes, was assisted by formulating the scattered spectrum as an expansion of normal modes. Conoir et al. ${ }^{6}$ focused in particular on the wave modes and resonant frequencies resulting from com-

\footnotetext{
a) Author to whom correspondence should be addressed; electronic mail: sinclair@mie.utoronto.ca
}

pression waves incident over a range of oblique angles $\alpha$. Comprehensive reviews of these topics and extensive bibliographies can be found in the works of Uberall, ${ }^{7}$ Pao and Mow, ${ }^{8}$ Gaunaurd, ${ }^{9}$ Uberall, ${ }^{10}$ and Veksler. ${ }^{11}$ Relatively few studies have been conducted on scattering from cylinders encased in a solid elastic matrix. Lewis and Kraft applied a normal-mode decomposition technique to this problem, although their solution is valid only in the long wavelength limit. ${ }^{12}$ Flax and Uberall ${ }^{13}$ conducted numerical studies on the interfacial waves that arise when compression waves are incident on spherical iron inclusions inside a solid aluminum matrix; experimental verification of their results was obtained only recently, ${ }^{14}$ and included a brief investigation on Stoneley waves on the matrix/inclusion interface. Hsieh et al. noted that the propagation of Stoneley waves along an interface is sensitive to several boundary parameters, such as bond integrity and degree of contact. ${ }^{15}$ Beattie et al. obtained reasonable agreement between theory and experiment for plane waves normally incident on isotropic rods embedded in epoxy. ${ }^{16}$

Possible application of this line of investigation was pursued by Sinclair and Addison, who compared the experimental and theoretical diffraction spectrum of a $\mathrm{SiC}$ fiber in a titanium matrix ${ }^{17}$ similar studies were later conducted for steel wires in plastic and included the cases of both good and bad interfacial adhesion. ${ }^{18}$ In both sets of studies, only normally incident compression waves were employed, such that the transversely isotropic nature of the scattering cylinder did not play a role. Huang et al. suggested the incorporation of a spring boundary condition to represent the condition of the interface in numerical modeling. ${ }^{19,20}$ Honarvar and Sinclair 


\section{Plane Wave Front}

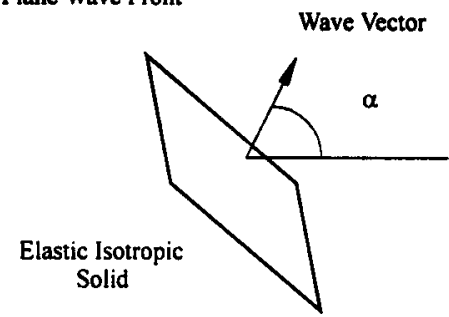

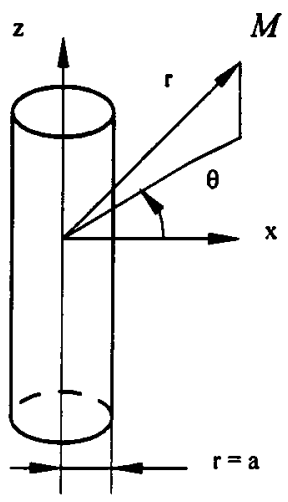

FIG. 1. Infinite plane wave incident at an angle $\alpha$ on a transversely isotropic cylinder embedded in a solid isotropic matrix. The observation point is $M(r, \theta, z)$.

proposed a systematic method to extract elastic constants from the diffracted spectrum resonances, called Material Characterization by Resonance Acoustic Spectroscopy (MCRAS). ${ }^{21}$

Many manufactured rods, fibers, and other cylindrical structures have transverse isotropy. There has been considerable study of acoustic wave-guide propagation along such structures, e.g., Refs. 22 and 23, but relatively little work on acoustic scattering from transversely isotropic cylindrical structures. Experimental measurements on scattering spectra from anisotropic cylinders in water were reported by de Billy ${ }^{24,25}$ More recently, Honarvar and Sinclair developed an exact normal-mode expansion for the scattering of a compression wave from an immersed, transversely isotropic cylinder, ${ }^{26}$ and compared theoretical to measured spectra for the case of a fiber-reinforced rod with high axial stiffness. ${ }^{21}$ The extension to be pursued here to a transversely isotropic cylinder encased in an elastic matrix adds considerable complication; now shear components of various polarizations must be included in both the incident and scattered waveform.

\section{THEORY}

\section{A. Displacement potential functions}

Consider a plane wave traveling through an infinite, isotropic medium, incident at an angle $\alpha$ on a long, transversely isotropic cylinder of radius $r=a$ (Fig. 1). The cylinder may be either a stiff rod, or a flexible reinforcing fiber encased in a matrix. The displacement field $\mathbf{u}(r, \theta, z, t)$ in both the matrix and cylinder can be expressed in terms of three scalar potentials $\phi, \psi$, and $\chi,{ }^{26}$

$$
\mathbf{u}=\nabla \phi+\nabla \times\left(\chi \mathbf{e}_{z}\right)+a \nabla \times \nabla \times\left(\psi \mathbf{e}_{z}\right) .
$$

For the case $\alpha=0$, the three potential functions in Eq. (1) correspond to compressional, transversely ( $r-\theta$ plane) polarized shear, and axially polarized shear components, respectively; such a tidy decomposition of Eq. (1) into constituent parts is not possible for nonzero values of incident angle $\alpha$.

In the matrix material, each of the three wave potentials can have two components: an "incident" plane wave component, and a "scattered" component corresponding to the field originating from the cylinder. The method of solution is

to first develop general expressions for the wave potentials in both the cylinder (material 1) and matrix (material 2), by substituting Eq. (1) into the equation of motion. Values for the unknown coefficients in the potentials are then determined by applying the appropriate boundary conditions at the surface of the embedded cylinder.

For the case where the incident wave is compressional with circular frequency $\omega$, the associated normalized potential function in the matrix (material 2) has the general form, ${ }^{17}$

$\phi_{2, \text { incident }}=\sum_{n=0}^{\infty} \epsilon_{n}(i)^{n} J_{n}\left(K_{2 \perp} r\right) \cos (n \theta) \exp \left(i\left[K_{2 z} z-\omega t\right]\right)$,

where $K_{2 \perp}=K_{2} \cos \alpha, K_{2 z}=K_{2} \sin \alpha$, and $K_{2}$ is the compressional wave number in the matrix material. The symbol $\epsilon_{n}$ is the Neumann factor $\left(\epsilon_{n}=1\right.$ for $n=0$, and $\epsilon_{n}=2$ for $n$ $>0$ ). The $J_{n}$ terms are Bessel functions of the first kind. For a shear wave polarized in the $r-\theta$ plane, the associated normalized potential is

$\chi_{2, \text { incident }}=\sum_{n=0}^{\infty} \epsilon_{n}(i)^{n} J_{n}\left(k_{2 \perp} r\right) \sin (n \theta) \exp \left(i\left[k_{2 z} z-\omega t\right]\right)$,

where $k_{2 \perp}=k_{2} \cos \alpha, k_{2 z}=k_{2} \sin \alpha$, and $k_{2}$ is the shear wave number in the matrix. Last, for the case of an incident shear wave polarized in the $r-z$ plane, the associated potential function is ${ }^{16}$

$\psi_{2, \text { incident }}=\sum_{n=0}^{\infty} \epsilon_{n}(i)^{n} J_{n}\left(k_{2 \perp} r\right) \cos (n \theta) \exp \left(i\left[k_{2 z} z-\omega t\right]\right)$.

Substitution of the appropriate combination of Eqs. (2), (3), and (4) into Eq. (1) then yields the total displacement field $\mathbf{u}_{2, \text { incident }}$ associated with the incident wave.

Consider now the transversely isotropic cylinder (material 1) of density $\rho_{1}$, where the constitutive relationship is characterized by five independent elastic constants,

$$
\begin{aligned}
{\left[\begin{array}{c}
\sigma_{r r} \\
\sigma_{\theta \theta} \\
\sigma_{z z} \\
\sigma_{\theta z} \\
\sigma_{r z} \\
\sigma_{r \theta}
\end{array}\right] } & {\left[\begin{array}{cccccc}
c_{11} & c_{12} & c_{13} & 0 & 0 & 0 \\
c_{12} & c_{11} & c_{13} & 0 & 0 & 0 \\
c_{13} & c_{13} & c_{33} & 0 & 0 & 0 \\
0 & 0 & 0 & c_{44} & 0 & 0 \\
0 & 0 & 0 & 0 & c_{44} & 0 \\
0 & 0 & 0 & 0 & 0 & \frac{\left(c_{11}-c_{12}\right)}{2}
\end{array}\right] } \\
& \times\left[\begin{array}{c}
\epsilon_{r r} \\
\epsilon_{\theta \theta} \\
\epsilon_{z z} \\
2 \epsilon_{\theta z} \\
2 \epsilon_{r z} \\
2 \epsilon_{r \theta}
\end{array}\right] .
\end{aligned}
$$

The displacement field $\mathbf{u}_{1}$ for $r<a$ can also be expressed in the format of Eq. (1). The associated potential functions required to give a nontrivial solution are given by ${ }^{26}$ 


$$
\begin{aligned}
\phi_{1}= & \sum_{n=0}^{\infty}\left[A_{n} J_{n}\left(s_{1} r\right)+q_{2} B_{n} J_{n}\left(s_{2} r\right)\right] \\
& \times \cos (n \theta) \exp \left(i\left[\kappa_{z} z-\omega t\right]\right), \\
\psi_{1}= & \sum_{n=0}^{\infty}\left[q_{1} A_{n} J_{n}\left(s_{1} r\right)+B_{n} J_{n}\left(s_{2} r\right)\right] \\
& \times \cos (n \theta) \exp \left(i\left[\kappa_{z} z-\omega t\right]\right), \\
\chi_{1}= & \sum_{n=0}^{\infty} C_{n} J_{n}\left(s_{3} r\right) \sin (n \theta) \exp \left(i\left[\kappa_{z} z-\omega t\right]\right),
\end{aligned}
$$

where the unknown coefficients $A_{n}, B_{n}$, and $C_{n}$ have yet to be determined. Continuity of stress and displacement at the $\mathrm{rod} / \mathrm{matrix}$ interface dictate that the axial wave vector $\kappa_{z}$ inside the rod must be equal to the axial wave vector outside of the rod: $K_{2 z}$ for the case of an incident compression wave, or $k_{2 z}$ in the case of an incident shear wave. It is noted that Eqs. (6a) and (6b) are coupled together, while Eq. (6c) is uncoupled. (This is analogous to the coupling between a compression wave and vertically polarized shear wave inside a plate, while the horizontally polarized shear wave is uncoupled.) The wave numbers $s_{1}, s_{2}$, and $s_{3}$ are given by

$$
\begin{aligned}
& s_{1}^{2}=\frac{\xi-\sqrt{\xi^{2}-4 \zeta c_{11} c_{44}}}{2 c_{11} c_{44}}, \\
& s_{2}^{2}=\frac{\xi+\sqrt{\xi^{2}-4 \zeta c_{11} c_{44}}}{2 c_{11} c_{44}}, \\
& s_{3}^{2}=\frac{2\left(\rho_{1} \omega^{2}-c_{44} \kappa_{z}^{2}\right)}{c_{11}-c_{12}},
\end{aligned}
$$

where

$$
\xi=\left(c_{13}+c_{44}\right)^{2} \kappa_{z}^{2}+c_{11}\left(\rho_{1} \omega^{2}-c_{33} \kappa_{z}^{2}\right)+c_{44}\left(\rho_{1} \omega^{2}-c_{44} \kappa_{z}^{2}\right)
$$

and

$$
\zeta=\left(\rho_{1} \omega^{2}-c_{44} \kappa_{z}^{2}\right)\left(\rho_{1} \omega^{2}-c_{33} \kappa_{z}^{2}\right) .
$$

The parameters $q_{1}$ and $q_{2}$ appearing in Eq. (6) are given by

$$
\begin{aligned}
& q_{1}=-\frac{-c_{11} s_{1}^{2}-\left(c_{13}+2 c_{44}\right) \kappa_{z}^{2}+\rho_{1} \omega^{2}}{a i \kappa_{z}\left[-\left(c_{11}-c_{13}-c_{44}\right) s_{1}^{2}-c_{44} \kappa_{z}^{2}+\rho_{1} \omega^{2}\right]}, \\
& q_{2}=-\frac{a i \kappa_{z}\left[-\left(c_{11}-c_{13}-c_{44}\right) s_{2}^{2}-c_{44} \kappa_{z}^{2}+\rho_{1} \omega^{2}\right]}{-c_{11} s_{2}^{2}-\left(c_{13}+c_{44} \kappa_{z}^{2}+\rho_{1} \omega^{2}\right)} .
\end{aligned}
$$

Last, one must consider the component of the displacement field $\mathbf{u}$ in the matrix material originating from waves scattered by the cylinder, and the associated potential functions, ${ }^{17}$

$$
\begin{aligned}
& \phi_{2, \text { scattered }}=\sum_{n=0}^{\infty} D_{n} H_{n}\left(K_{2 \perp} r\right) \cos (n \theta) \exp \left(i\left[\kappa_{z} z-\omega t\right]\right), \\
& \psi_{2, \text { scattered }}=\sum_{n=0}^{\infty} E_{n} H_{n}\left(k_{2 \perp} r\right) \cos (n \theta) \exp \left(i\left[\kappa_{z} z-\omega t\right]\right),
\end{aligned}
$$

$$
\chi_{2, \text { scattered }}=\sum_{n=0}^{\infty} F_{n} H_{n}\left(k_{2 \perp} r\right) \sin (n \theta) \exp \left(i\left[\kappa_{z} z-\omega t\right]\right),
$$

where $H_{n}$ represents the Hankel function of the first kind. [Although Hankel functions of both the first and second kind satisfy the wave equation in cylindrical coordinates, only the former are consistent with the condition of an outwardly propagating wave, scattered from the rod. ${ }^{27}$ It is noted that the expressions of Eq. (6) for the wave potentials inside the rod were given in terms of Bessel functions of the first kind, $J_{n}$, because Hankel functions become infinite as their argument approaches zero.] The total displacement in material 2 can now be obtained by summing the incident and scattered components,

$$
u_{2}=u_{2, \text { incident }}+u_{2, \text { scattered }}
$$

and making the appropriate substitutions from Eqs. (1)-(4) and (12).

\section{B. Boundary conditions}

There are six boundary conditions related to the continuity of stress and displacement at the cylinder/matrix interface,

$$
\begin{aligned}
& {\left[u_{r}, u_{\theta}, u_{z}, \sigma_{r r}, \sigma_{r \theta}, \sigma_{r z}\right]_{1}} \\
& \quad=\left[u_{r}, u_{\theta}, u_{z}, \sigma_{r r}, \sigma_{r \theta}, \sigma_{r z}\right]_{2} \text { at } r=a,
\end{aligned}
$$

where expressions for the stress at any point can be derived from the displacement field determined according to Eq. (1), plus the strain-displacement relationships and Hooke's Law. In matrix format, Eq. (14) can be rewritten as

$$
\left[\begin{array}{llllll}
a_{11} & a_{12} & a_{13} & a_{14} & a_{15} & a_{16} \\
a_{21} & a_{22} & a_{23} & a_{24} & a_{25} & a_{26} \\
a_{31} & a_{32} & a_{33} & a_{34} & a_{35} & a_{36} \\
a_{41} & a_{42} & a_{43} & a_{44} & a_{45} & a_{46} \\
a_{51} & a_{52} & a_{53} & a_{54} & a_{55} & a_{56} \\
a_{61} & a_{62} & a_{63} & a_{64} & a_{65} & a_{66}
\end{array}\right]\left[\begin{array}{c}
A_{n} \\
B_{n} \\
C_{n} \\
D_{n} \\
E_{n} \\
F_{n}
\end{array}\right]=\left[\begin{array}{c}
b_{1} \\
b_{2} \\
b_{3} \\
b_{4} \\
b_{5} \\
b_{6}
\end{array}\right],
$$

where the $a_{i j}$ and $b_{i}$ coefficients are given in the Appendix. Solution of Eq. (15) followed by substitution into Eqs. (1), (5), and (12), allows the calculation of the normalized spectrum of scattered waves, also known as the backscattered form function $f_{\infty}$. For the case of incident compression waves, it is given by

$$
f_{\infty}\left(K_{2} a\right)=\sum_{n}\left(2 / \sqrt{i \pi K_{2} a}\right)(-1)^{n} \epsilon_{n} Q_{n},
$$

and for incident shear waves (of either $r-z$ or $r$ - $\theta$ polarization) by

$$
f_{\infty}\left(k_{2} a\right)=\sum_{n}\left(2 / \sqrt{i \pi k_{2} a}\right)(-1)^{n} \epsilon_{s n} Q_{n} .
$$

The parameter $Q_{n}$ depends on the type of scattered wave of interest, as defined in Eq. (12): $D_{n}, E_{n}$, or $F_{n}$, corresponding to scattered compression waves, $r-z$ polarized shear waves, or $r-\theta$ polarized shear waves, respectively. 
TABLE I. Properties of isotropic materials.

\begin{tabular}{lcrcc}
\hline \hline \multicolumn{1}{c}{ Material } & Density $\left(\mathrm{kg} / \mathrm{m}^{3}\right)$ & $\lambda(\mathrm{GPa})$ & $\mu(\mathrm{GPa})$ & \multirow{2}{*}{$\begin{array}{c}\text { Lame constants } \\
(\mathrm{GPa})\end{array}$} \\
\hline Water & 1000 & & 0 & 2.2 \\
Stainless steel $^{\mathrm{a}}$ & 9449 & 140.2 & 93.8 & \\
Epoxy $^{\mathrm{b}}$ & 1129 & 3.9 & 2.0 & \\
\hline \hline
\end{tabular}

${ }^{\mathrm{a} F r o m ~ R e f . ~} 21$

${ }^{\mathrm{b}}$ From Ref. 16.

\section{NUMERICAL SIMULATION}

The MATLAB software library was selected to solve Eq. (15) for any user-specified array of input parameters: elastic constants of matrix and cylinder, cylinder radius, incidence angle $\alpha$, frequency span, and frequency step. One may also specify the mode and polarization of both the incident wave and scattered wave of interest: compression, shear polarized in the $r-z$ plane, or shear polarized in the transverse $(r-\theta)$ plane. A graphical user interface (GUI) is used for managing the data input and results. The program is implemented on a Pentium II processor operating at 350 MHz. Partial verification of this new model is possible by comparing the results of a restricted class of test cases with those published in the literature; two such examples are indicated in the following paragraphs. A rigorous verification of the new formulation by this route is not possible, as there are limited published results corresponding to the selection of wave modes, incidence angles, and material properties encompassed by Eq. (15).

The first test case is that of an axially polarized shear wave normally incident on a $0.37-\mathrm{mm}$ radius stainless steel wire embedded in an isotropic epoxy matrix. Elastic properties of the materials are given in Table I. The form function $f_{\infty}$ of the scattered axially polarized shear wave, indicated by the solid line in Fig. 2, is identical to that obtained by Beattie et al. ${ }^{16}$ although their formulation did not include the op-

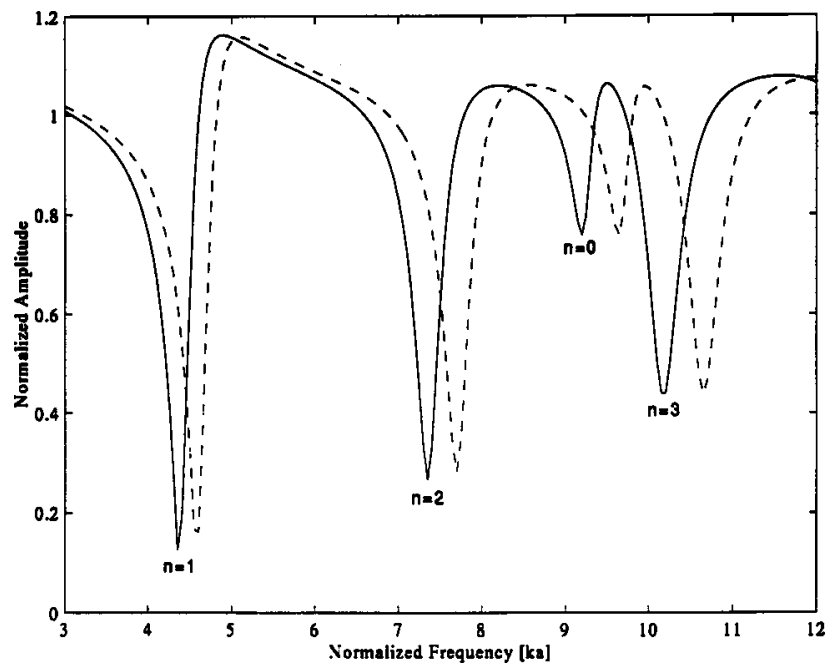

FIG. 2. Form function of the backscattered axially polarized shear wave, resulting from an axially polarized shear wave incident at $\alpha=0 \mathrm{deg}$ on a cylinder of radius $0.37 \mathrm{~mm}$. The cylinder is embedded in an isotropic epoxy matrix. Solid line: isotropic stainless steel cylinder; dashed line: stainless steel cylinder, but with a $10 \%$ increase in $c_{44}\left(=c_{55}\right)$, with all other stiffness constants left unchanged.

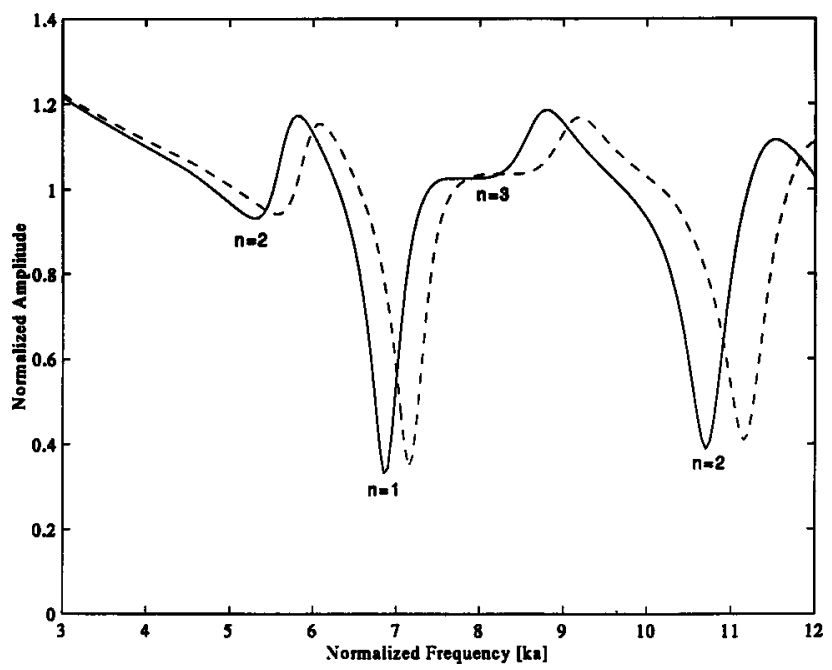

FIG. 3. Form function of backscattered shear waves polarized in the $r-\theta$ plane, resulting from $r$ - $\theta$ polarized shear wave incident at $\alpha=0 \mathrm{deg}$. The cylinder is embedded in an isotropic epoxy matrix. Solid line: Isotropic stainless steel cylinder; dashed line: Stainless steel cylinder, but with a $10 \%$ increase in $c_{66}$. [A corresponding adjustment was also made to the value of $c_{11}$, according to the relation $c_{66}=\left(c_{11}-c_{12}\right) / 2$.]

tions of a nonzero angle of incidence, nor that of a transversely isotropic rod. The major resonances correspond to an integral number $n=1,2,3$ of wavelengths of the axially polarized waves encircling the boundary of the cylinder; the integer $n$ for each resonance was identified via a decomposition of the normal-mode expansion series of Eq. (12) into its constituent parts. A higher order "breathing mode," corresponding to $n=0$, is also observed near $k a=9$.

The major resonances corresponding to $n=1,2,3, \ldots$ in Fig. 2 must correspond to a dispersive wave mode, as there is a nonlinear correlation between $n$ and normalized frequency $k a$. This dispersion is due to the finite curvature of the rod, which leads to significant penetration of the wave mode through the rod $(\lambda / a$ is of the order 1) for small values of $n$. The dispersion disappears at very large values of $n$ corresponding to high frequencies and short wavelength; in this limit, the numerical simulation indicates a characteristic wave velocity of approximately $3500 \mathrm{~m} / \mathrm{s}$.

Also shown in Fig. 2 is the effect on $f_{\infty}$ of switching from an isotropic to a transversely isotropic rod by increasing the shear modulus $c_{44}\left(=c_{55}\right)$ by $10 \%$, while leaving all other stiffness constants unchanged. This would have the effect of increasing the velocity of axially polarized bulk shear waves by about 5\%; a corresponding shift in the resonance frequencies is noted in Fig. 2.

Figure 3 show the form function for the identical rod/ matrix system of Fig. 2, but corresponding to incident and scattered shear waves polarized in the $r$ - $\theta$ plane, with $\alpha$ $=0$. Mode conversion leads to the presence of both compressional and shear components inside the rod, although axially polarized shear components (corresponding to the potential $\psi)$ are absent. Within the frequency range spanned in Fig. 3, two pronounced resonances are evident $(n=1,2)$, corresponding to waves encircling the rod along the rod-epoxy interface. The resonance frequencies $f_{R}$ are closely linked to the transverse shear modulus $c_{66}$; this relationship leads to 

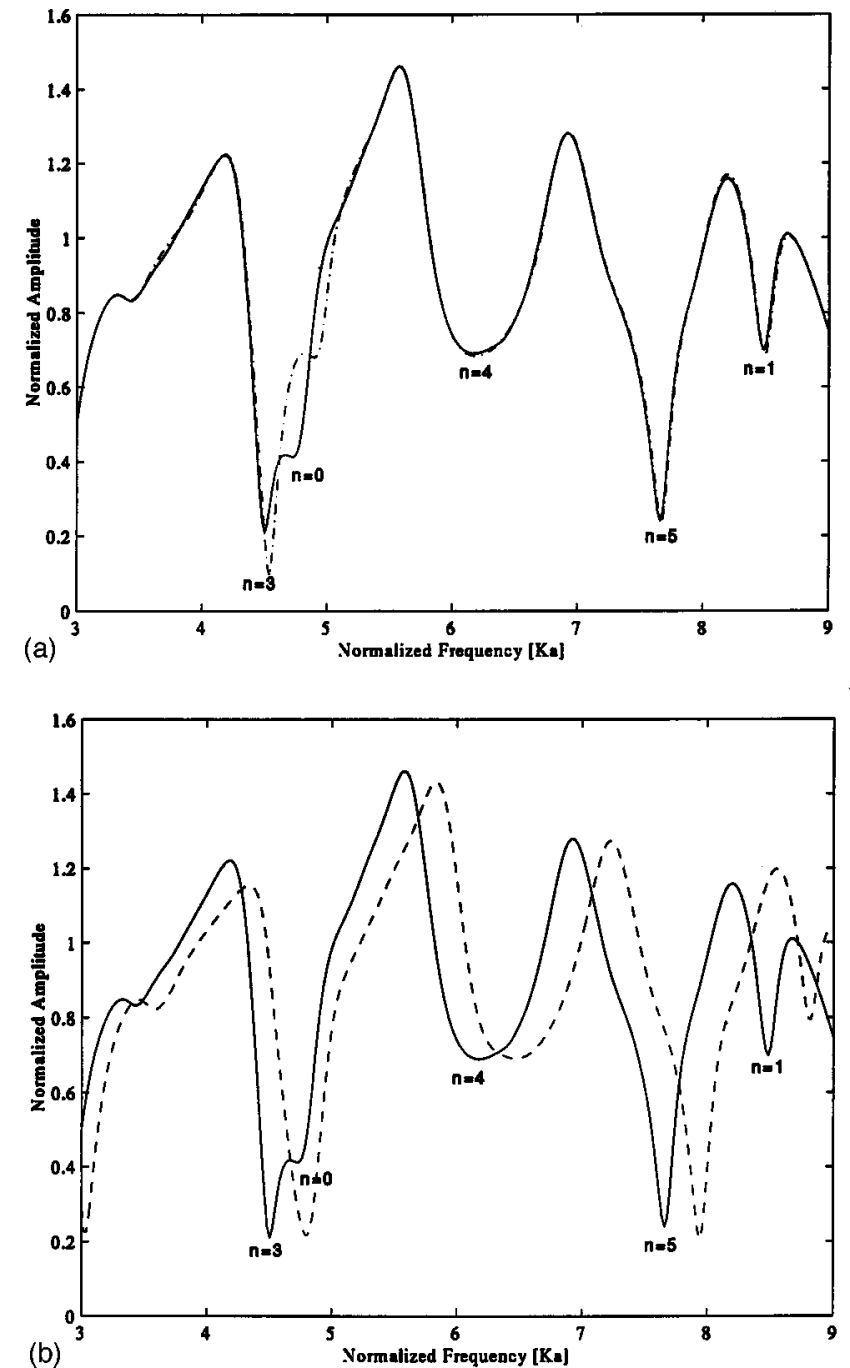

FIG. 4. Form function of the compression wave scattered at $\theta=180 \mathrm{deg}$, resulting from a plane compression wave incident at an angle $\alpha=3 \mathrm{deg}$. The cylinder is embedded in an isotropic epoxy matrix. Only one of the resonances, corresponding to $n=0$ corresponds to a guided mode. (a) Compression wave scattered at $\theta=180 \mathrm{deg}$; Solid line: isotropic stainless steel cylinder; dashed line: stainless steel cylinder with a $10 \%$ increase in $c_{44}$ $\left(=c_{55}\right.$ ), with all other stiffness constants left unchanged. (b) Compression wave scattered at $\theta=180 \mathrm{deg}$ : Solid line: isotropic stainless steel cylinder; dashed line: stainless steel cylinder with a $10 \%$ increase in $c_{66}$. [A corresponding adjustment was also made to the value of $c_{11}$, according to the relation $\left.c_{66}=\left(c_{11}-c_{12}\right) / 2.\right]$

the expected positive correlation between $f_{R}$ and $c_{66}$ for both resonances, as shown in Fig. 3.

Figure 4 features the same isotropic rod/matrix system as in Figs. 2 and 3, but now for a plane compression wave incident at an angle $\alpha=3^{\circ}$. This incident wave leads to scattered compression waves, and shear waves with components polarized along both the $z$-axis and in the $r-\theta$ planes. Figure 4(a) shows $f_{\infty}$ corresponding to compression waves backscattered at $\theta=180 \mathrm{deg}$. Due to the small value of the incidence angle $\alpha$, the resonances are primarily associated with waves encircling the rod, as was the case in Figs. 2 and 3. Only one axially guided mode is observed, corresponding to $n=0$, at $k a \approx 4.8$. The differing sensitivities of these two types of resonances to perturbations in the elastic constants of the rod are quite striking: a $10 \%$ increase in $c_{44}\left(=c_{55}\right)$,
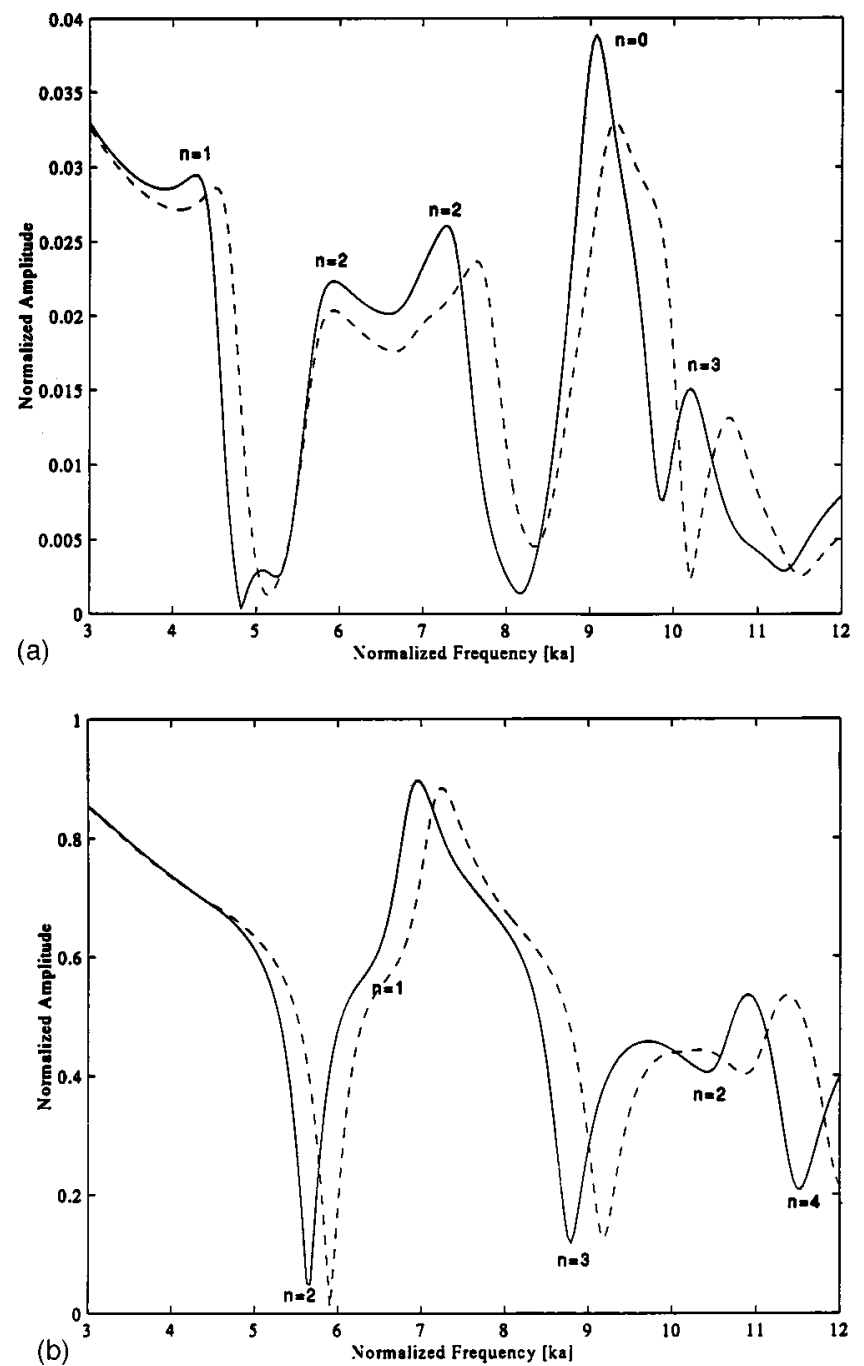

FIG. 5. Form function corresponding to scattered shear waves, resulting from a plane compression wave incident at an angle $\alpha=3 \mathrm{deg}$. The cylinder is embedded in an isotropic epoxy matrix. (a) Axially polarized shear waves scattered at $\theta=180 \mathrm{deg}$ : Solid line: isotropic stainless steel cylinder; dashed line: stainless steel cylinder with a $10 \%$ increase in $c_{44}\left(=c_{55}\right)$, with all other stiffness constants left unchanged. (b) Transversely polarized shear wave scattered at $\theta=180 \mathrm{deg}$ : Solid line: isotropic stainless steel cylinder; dashed line: stainless steel cylinder with a $10 \%$ increase in $c_{66}$. [A corresponding adjustment was also made to the value of $c_{11}$, according to the relation $c_{66}=\left(c_{11}-c_{12}\right) / 2$.]

shown by the dashed line in Fig. 4(a) leads to an upward shift in $f_{R}$ for the guided mode resonance, which is dominated by shearing motion in the $\theta-z$ and $r-z$ planes. However, there is no shift in $f_{R}$ corresponding to any of the other resonances, which are dominated by strain in the $r-\theta$ plane.

In Fig. 4(b), it is seen that a $10 \%$ increase in $c_{66}$ for the rod leads to an increase in $f_{R}$ for all resonances, except for the guided mode. In fact, the guided mode resonance is no longer visible in the form function when $c_{66}$ is increased, as the resonance dip is masked by the strong interfacial mode resonance corresponding to $n=3$.

Figure 5(a) shows the form function corresponding to axially polarized shear waves scattered from the cylinder, as a result of compression waves incident at an angle $\alpha$ $=3 \mathrm{deg}$. The small amplitude of $f_{\infty}$ in Fig. 5(a) is noteworthy—only a small component of the molecular mo- 
tion in the incident compression wave is polarized in the axial direction due to the small angle of incidence $\alpha$. (There are considerable experimental difficulties in the measurement of form functions for large $\alpha$ values.) A second interesting feature of this graph is that the resonances appear as peaks, instead of the dips seen in Figs. 2-4; this is due to a phase matching between the energy leaked from the resonant mode and the main echo backscattered from the cylindermatrix interface. ${ }^{28,29}$

It has been reported that the shearing components of the cylinder/matrix boundary conditions [Eq. (14)] may be particularly sensitive to imperfections in the interface. ${ }^{30,31} \mathrm{~A}$ defective interface would therefore be most apparent in the form functions corresponding to modes featuring shearing action at the boundaries, and transmission of shear waves through the interface into the matrix. These considerations have formed the basis for NDE techniques to characterize fiber-reinforced composite materials, utilizing selected modes of axially guided waves. Consistent with this premise, it is seen in Fig. 5(a) that the form function is sensitive to $c_{44}\left(=c_{55}\right)$, in a manner similar to that for the axially polarized shear wave normally incident on the stainless steel rod seen in Fig. 2. However, the relatively weak signal, as indicated by the vertical scale of Fig. 5(a), would greatly hinder the use of this particular setup in a NDE scheme for such a small incidence angle $\alpha$.

The form function of Fig. 5(b) corresponding to scattered shear waves polarized in the $r-\theta$ plane has a higher amplitude than that of Fig. 5(a). The resonance frequencies are seen to have good sensitivity to perturbations in the value of $c_{66}$ for the cylinder; experimental work is required to determine whether a weak interface correlates more closely with perturbations in $c_{66}$ or in $c_{44}$. The contrast between Figs. 5(b) and 3 is striking: both feature the form function corresponding to scattered shear wave components in the $r$ - $\theta$ plane. Although the shapes of the two form functions appear markedly different, closer examination reveals that the resonant frequencies in the two figures are very similar. It is primarily the oscillatory background components of the two figures that are so different; this background is particularly sensitive to the incidence angle $\alpha$.

Figure 6(a) shows the form function corresponding to scattered compression waves, resulting from compression waves obliquely incident at an angle $\alpha=3^{\circ}$ on a transversely isotropic rod immersed in water. [The horizontal axis is expressed in units of $\mathrm{MHz}$, in order to facilitate comparison with Fig. 6(b)-(d).] The rod is a continuous-fiber aluminum matrix composite (CF-AMC) manufactured by $3 \mathrm{M}$, with elastic properties given in Table II. The form function is identical to that reported earlier by Honarvar and Sinclair. ${ }^{21}$ The spectrum features leaky pseudo-Rayleigh $(R)$ resonances at $f=0.95$ and $1.45 \mathrm{MHz}$; whispering gallery (WG) modes at $f=0.8$ and $1.3 \mathrm{MHz}$; and a leaky axially guided (GD) mode at $f=1.2 \mathrm{MHz}$. These are all superimposed on an oscillating background originating from Franz and Scholte-Stoneley waves in the fluid encircling the rod. It should be noted that the water imparts minimal loading on the rod, such that the resonances appearing in Fig. 6(a) represent close to free modes of vibration.
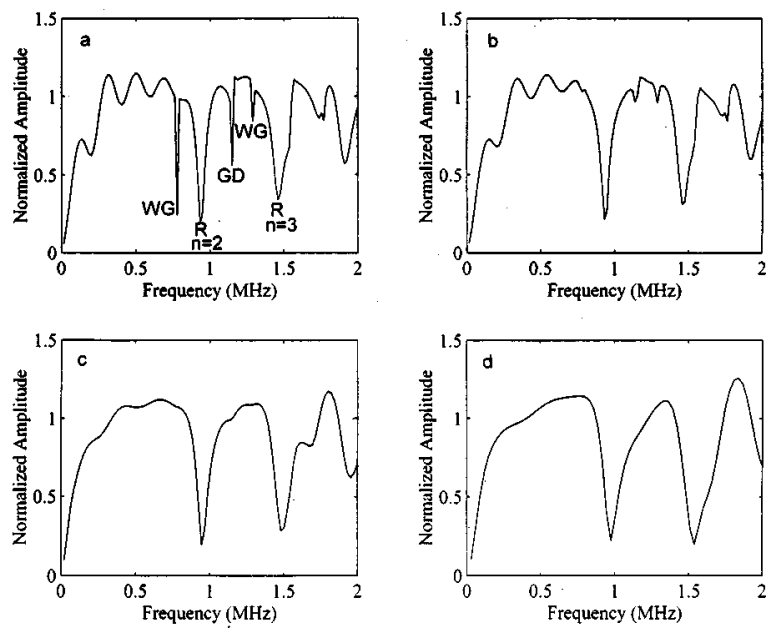

FIG. 6. Form functions corresponding to backscattered compression waves, resulting from a compression wave obliquely incident at an angle $\alpha$ $=3 \mathrm{deg}$ on a transversely isotropic CF-AMC rod. The moduli of the surrounding medium are increased in steps from graph (a) to graph (d), where: For graph (a): Bulk modulus $=2.2 \mathrm{GPa} ; \mu=0$ (corresponding to water); for graph (d): $\lambda=3.9 \mathrm{GPa} ; \mu=2.0 \mathrm{GPa}$ (corresponding to epoxy).

Figure 6(b) to (d) show the effects of step increases in the elastic constants of the bulk medium surrounding the rod: from values corresponding to water in Fig. 6(a), to those describing epoxy in Fig. 6(d). The oscillatory background, whispering gallery modes, and guided mode are all suppressed as the elastic constants of the matrix are increased in the sequence of simulations. The pseudo-Rayleigh resonances become associated with interfacial modes, although their resonant frequencies are shifted only to a marginal extent from their values in Fig. 6(a). The small size of this shift is believed to be due to the relatively low acoustic impedance of the epoxy compared to the CF-AMC. In the limit of a large radius-to-wavelength ratio, these interfacial modes are associated with leaky pseudo-Stoneley waves, ${ }^{32}$ and have been recommended for NDE of interfacial properties of adhesive bonds or interference-fit fasteners. ${ }^{30,31}$

The interfacial waves existing on the curved rod-matrix interface are dispersive. Lee and Corbly ${ }^{31}$ have determined dispersion curves for axially propagating Stoneley waves on a cylindrical interface; however, the experimental constraint of relatively small incidence angles $\alpha$ restricts our interest to wave vectors oriented primarily in the $r-\theta$ plane. It would be possible to construct dispersion curves for these interfacial waves by considering the family of resonances over a large frequency range, in a manner similar to that used by Flax and Uberall for spherical inclusions embedded in a solid matrix. $^{13}$

\section{CONCLUSION}

A normal-mode expansion solution has been presented for the wave scattered by a transversely isotropic cylinder embedded in a solid elastic medium; the formulation accommodates incident plane waves of compression mode, axially polarized shear, or $r-\theta$ polarized shear, at any angle of incidence $\alpha$ with respect to the axis of the rod. The geometry is a good model for a composite material featuring a reinforcing fiber encased in an elastic matrix. Results from the new 


\begin{tabular}{ccccccc}
\hline \hline Density $\left(\mathrm{kg} / \mathrm{m}^{3}\right)$ & $c_{11}(\mathrm{GPa})$ & $c_{33}(\mathrm{GPa})$ & $c_{44}(\mathrm{GPa})$ & $c_{13}(\mathrm{GPa})$ & $c_{12}(\mathrm{GPa})$ & $\begin{array}{c}c_{66}(\mathrm{GPa}) \\
=\left(c_{11}-c_{12}\right) / 2\end{array}$ \\
\hline 3220 & 179 & 309 & 50 & 95 & 84 & 47.7 \\
\hline \hline
\end{tabular}

${ }^{\mathrm{a}}$ Elastic constants estimated to within $\pm 3 \%$.

formulation indicate several resonances that are sensitive to perturbations in the elastic constants of the cylinder. Leaky pseudo-Stoneley waves that travel on the matrix-cylinder interface lead to resonances in the scattered wave spectrum that would be good indicators of deficiencies in the integrity of the interface. A Regge-pole plot of these resonances would lead to dispersion curves for these waves, as a function of the wavelength-to-radius ratio.

To adapt the formulation to the nondestructive evaluation of fiber-reinforced composites, it would need to be expanded to accommodate multiple fibers in the matrix. Preliminary work in this direction has been conducted by other researchers, e.g., Ref. 33, for waves normally incident on a random distribution of fibers in a solid matrix. In addition, it would be advantageous to address the experimental difficulties that preclude the use of large incidence angles for the planer wave impinging on the embedded cylinder.

\section{ACKNOWLEDGMENT}

The authors gratefully acknowledge the financial support of the Natural Sciences and Engineering Research Council (NSERC) of Canada.

\section{APPENDIX}

Elements of the $\left\{a_{i j}\right\}$ matrix of Eq. (15) are as follows:

$$
\begin{aligned}
& a_{11}=\left(1+i a q_{1} \kappa_{z}\right)\left[n J_{n}\left(s_{1} a\right)-s_{1} a J_{n+1}\left(s_{1} a\right)\right], \\
& a_{12}=\left(q_{2}+i a \kappa_{z}\right)\left[n J_{n}\left(s_{2} a\right)-s_{2} a J_{n+1}\left(s_{2} a\right)\right], \\
& a_{13}=n J_{n}\left(s_{3} a\right), \\
& a_{14}=-n H_{n}\left(K_{2 \perp} a\right)+K_{2 \perp} a H_{n+1}\left(K_{2 \perp} a\right), \\
& a_{15}=-i a \kappa_{z}\left[n H_{n}\left(k_{2 \perp} a\right)-k_{2 \perp} a H_{n+1}\left(k_{2 \perp} a\right)\right], \\
& a_{16}=-n H_{n}\left(k_{2 \perp} a\right), \\
& a_{21}=\left(-n-i n a \kappa_{z} q_{1}\right) J_{n}\left(s_{1} a\right), \\
& a_{22}=\left(-n q_{2}-i n a \kappa_{z}\right) J_{n}\left(s_{2} a\right), \\
& a_{23}=-n J_{n}\left(s_{3} a\right)+s_{3} a J_{n+1}\left(s_{3} a\right), \\
& a_{24}=n H_{n}\left(K_{2 \perp} a\right), \\
& a_{25}=i n a \kappa_{z} H_{n}\left(k_{2 \perp} r\right), \\
& a_{26}=n H_{n}\left(k_{2 \perp} a\right)-k_{2 \perp} a H_{n+1}\left(k_{2 \perp} a\right), \\
& a_{31}=\left(a s_{1}^{2} q_{1}+i \kappa_{z}\right) J_{n}\left(s_{1} a\right), \\
& a_{32}=\left(a s_{2}^{2}+i \kappa_{z} q_{2}\right) J_{n}\left(s_{2} a\right), \quad a_{33}=0, \\
& a_{34}=-i \kappa_{z} H_{n}\left(K_{2 \perp} a\right), \\
& a_{35}=-a k_{2 \perp}^{2} H_{n}\left(k_{2 \perp} a\right), \quad a_{36}=0,
\end{aligned}
$$

$$
\begin{aligned}
a_{41}= & {\left[c_{11}^{(1)}+i a \kappa_{z} q_{1}\left(c_{11}^{(1)}-c_{13}^{(1)}\right)\right]\left[\left(n^{2}-n-s_{1}^{2} a^{2}\right) J_{n}\left(s_{1} a\right)\right.} \\
& \left.+s_{1} a J_{n+1}\left(s_{1} a\right)\right]+\left[c_{12}^{(1)}+\left(c_{12}^{(1)}-c_{13}^{(1)}\right) i a \kappa_{z} q_{1}\right] \\
& \times\left[n J_{n}\left(s_{1} a\right)-s_{1} a J_{n+1}\left(s_{1} a\right)\right]+\left[-c_{13}^{(1)} \kappa_{z}^{2} a^{2}\right. \\
& \left.-c_{12}^{(1)} n^{2}+\left(c_{13}^{(1)}-c_{12}^{(1)}\right) i a n^{2} q_{1} \kappa_{z}\right] J_{n}\left(s_{1} a\right), \\
a_{42}= & {\left[c_{11}^{(1)} q_{2}+i a \kappa_{z}\left(c_{11}^{(1)}-c_{13}^{(1)}\right)\right]\left[\left(n^{2}-n-s_{2}^{2} a^{2}\right) J_{n}\left(s_{2} a\right)\right.} \\
& \left.+s_{2} a J_{n+1}\left(s_{2} a\right)\right]+\left[c_{12}^{(1)} q_{2}+\left(c_{12}^{(1)}-c_{13}^{(1)}\right) i a \kappa_{z}\right] \\
& \times\left[n J_{n}\left(s_{2} a\right)-s_{2} a J_{n+1}\left(s_{2} a\right)\right]+\left[-c_{13}^{(1)} \kappa_{z}^{2} q_{2} a^{2}\right. \\
& \left.-c_{12}^{(1)} n^{2} q_{2}+\left(c_{13}^{(1)}-c_{12}^{(1)}\right) i a n^{2} \kappa_{z}\right] J_{n}\left(s_{2} a\right), \\
a_{43}= & \left(c_{11}^{(1)}-c_{12}^{(1)}\right) n\left[(n-1) J_{n}\left(s_{3} a\right)-s_{3} a J_{n+1}\left(s_{3} a\right)\right], \\
a_{44}= & -\left[c_{11}^{(2)}\left(n^{2}-n-K_{2 \perp}^{2} a^{2}\right)+c_{12}^{(2)} n(1-n)\right. \\
& \left.-c_{13}^{(2)} \kappa_{z}^{2} a^{2}\right] H_{n}\left(K_{2 \perp} a\right) \\
& +\left(c_{12}^{(2)}-c_{11}^{(2)}\right) K_{2 \perp} a H_{n+1}\left(K_{2 \perp} a\right), \\
a_{55}= & \left(c_{11}^{(2)}-c_{12}^{(2)}\right)\left[H_{n}\left(k_{2 \perp} a\right) i a \kappa_{z} n(n-1)\right. \\
& \left.-i n \kappa_{z} k_{2 \perp} a^{2} H_{n+1}\left(k_{2 \perp} a\right)\right], \\
a_{54}= & \left(c_{11}^{(2)}-c_{12}^{(2)}\right)\left[H_{n}\left(K_{2 \perp} a\right) n(n-1)\right. \\
& \left.-n K_{2 \perp} a H_{n+1}\left(K_{2 \perp} a\right)\right], \\
a_{53}= & -i a \kappa_{z}\left(c_{11}^{(2)}-c_{13}^{(2)}\right)\left[\left(n^{2}-n-k_{2 \perp}^{2} a^{2}\right) H_{n}\left(k_{2 \perp} a\right)\right. \\
& \left.+k_{2 \perp} a H_{n+1}\left(k_{2 \perp} a\right)\right] \\
& -\left(c_{12}^{(2)}-c_{13}^{(2)}\right) i a \kappa_{z}\left[n H_{n}\left(k_{2 \perp} a\right)\right. \\
a_{52}= & n\left(c_{11}^{(1)}-c_{12}^{(1)}\right)\left(q_{2}+i a \kappa_{z}\right)\left[(1-n) J_{n}\left(s_{2} a\right)\right. \\
& \left.-k_{2 \perp} a H_{n+1} a^{2}-2 n(n-1)\right] J_{n}\left(s_{3} a\right) \\
& -\left(c_{13}^{2}-c_{12}^{2}\right) i a n^{2} \kappa_{z} H_{n}\left(k_{2 \perp} a\right), \\
a_{46}= & -\left(c_{11}^{(2)}-c_{12}^{(2)}\right) n\left[(n-1) H_{n}\left(k_{2 \perp} a\right)\right. \\
& \left.-k_{2 \perp} a H_{n+1}\left(k_{2 \perp} a\right)\right], \\
a_{51}= & n\left(c_{11}^{(1)}-c_{12}^{(1)}\right)\left(1+i a q_{1} \kappa_{z}\right)\left[(1-n) J_{n}\left(s_{1} a\right)\right. \\
& \left.s_{1} a J_{n+1}\left(s_{1} a\right)\right], \\
& \\
&
\end{aligned}
$$




$$
\begin{aligned}
a_{56}= & \frac{\left(c_{11}^{(2)}-c_{12}^{(2)}\right)}{2}\left[H_{n}\left(k_{2 \perp} a\right)\left(2 n(n-1)-k_{2 \perp}^{2} a^{2}\right)\right. \\
& \left.+2 k_{2 \perp} a H_{n+1}\left(k_{2 \perp} a\right)\right], \\
a_{61}= & c_{44}^{(1)}\left[q_{1}\left(s_{1}^{2} a-\kappa_{z}^{2} a\right)+2 i \kappa_{z}\right] \\
& \times\left[n J_{n}\left(s_{1} a\right)-s_{1} a J_{n+1}\left(s_{1} a\right)\right], \\
a_{62}= & c_{44}^{(1)}\left(s_{2}^{2} a-a \kappa_{z}^{2}+2 i \kappa_{z} q_{2}\right) \\
& \times\left[n J_{n}\left(s_{2} a\right)-s_{2} a J_{n+1}\left(s_{2} a\right)\right], \\
a_{63}= & c_{44}^{(1)} i n \kappa_{z} J_{n}\left(s_{3} a\right), \\
a_{64}= & c_{44}^{(2)}\left[-2 i n \kappa_{z} H_{n}\left(K_{2 \perp} a\right)\right. \\
& \left.+2 i \kappa_{z} K_{2 \perp} a H_{n+1}\left(K_{2 \perp} a\right)\right], \\
a_{65}= & c_{44}^{(2)}\left(\kappa_{z}^{2}-k_{2 \perp}^{2}\right)\left[a n H_{n}\left(k_{2 \perp} a\right)\right. \\
& \left.-k_{2 \perp} a^{2} H_{n+1}\left(k_{2 \perp} a\right)\right], \\
a_{66}= & -c_{44}^{(2)} i n \kappa_{z} H_{n}\left(k_{2 \perp} a\right) .
\end{aligned}
$$

Elements of the $\left\{b_{i}\right\}$ vector of Eq. (15) depend on the type of wave incident on the cylinder. For an incident compression wave, they are as follows:

$$
\begin{aligned}
b_{1}= & \epsilon_{n}(i)^{n}\left[n J_{n}\left(K_{2 \perp} a\right)-K_{2 \perp} a J_{n+1}\left(K_{2 \perp} a\right)\right], \\
b_{2}= & -n \epsilon_{n}(i)^{n} J_{n}\left(K_{2 \perp} a\right), \\
b_{3}= & \epsilon_{n}(i)^{n+1} \kappa_{z} J_{n}\left(K_{2 \perp} a\right), \\
b_{4}= & \epsilon_{n}(i)^{n}\left[c_{11}^{(2)}\left(n^{2}-n-K_{2 \perp}^{2} a^{2}\right)+c_{12}^{(2)} n(1-n)\right. \\
& \left.-c_{13}^{(2)} K_{2 \perp}^{2} a^{2}\right] J_{n}\left(K_{2 \perp} a\right) \\
& -\epsilon_{n}(i)^{n}\left(c_{12}^{(2)}-c_{11}^{(2)}\right) K_{2 \perp} a J_{n+1}\left(K_{2 \perp} a\right), \\
b_{5}= & \epsilon_{n}(i)^{n}\left(c_{11}^{(2)}-c_{12}^{(2)}\right)\left[J_{n}\left(K_{2 \perp} a\right)\left(n-n^{2}\right)\right. \\
& \left.+n K_{2 \perp} a J_{n+1}\left(K_{2 \perp} a\right)\right], \\
b_{6}= & c_{44}^{(2)} 2 \epsilon_{n}(i)^{n+1} \kappa_{z}\left[n J_{n}\left(K_{2 \perp} a\right)-K_{2 \perp} a J_{n+1}\left(K_{2 \perp} a\right)\right] .
\end{aligned}
$$

For an incident shear wave, polarized in the $r$ - $\theta$ plane,

$$
\begin{aligned}
b_{1}= & \epsilon_{n}(i)^{n} n J_{n}\left(k_{2 \perp} a\right), \\
b_{2}= & -\epsilon_{n}(i)^{n}\left(n J_{n}\left(k_{2 \perp} a\right)-k_{2 \perp} a J_{n+1}\left(k_{2 \perp} a\right)\right), \\
b^{3}= & 0, \\
b_{4}= & \epsilon_{n}(i)^{n}\left(c_{11}^{(2)}-c_{12}^{(2)}\right) n\left[(n-1) J_{n}\left(k_{2 \perp} a\right)\right. \\
& \left.-k_{2 \perp} a J_{n+1}\left(k_{2 \perp} a\right)\right], \\
b_{5}= & \epsilon_{n}(i)^{n} \frac{\left(c_{12}^{(2)}-c_{11}^{(2)}\right)}{2}\left[J_{n}\left(k_{2 \perp} a\right)\left(2 n(n-1)-k_{2 \perp}^{2} a^{2}\right)\right. \\
& \left.+2 k_{2 \perp} a J_{n+1}\left(k_{2 \perp} a\right)\right], \\
b_{6}= & \epsilon_{n}(i)^{n} c_{44}^{(2)} i n \kappa_{z} J_{n}\left(k_{2 \perp} a\right) .
\end{aligned}
$$

For an incident shear wave, polarized in the $r-z$ plane,

$$
\begin{aligned}
& b_{1}=\epsilon_{n}(i)^{n+1} a \kappa_{z}\left[n J_{n}\left(k_{2 \perp} a\right)-k_{2 \perp} a J_{n+1}\left(k_{2 \perp} a\right)\right], \\
& b_{2}=-\epsilon_{n}(i)^{n+1} n a \kappa_{z} J_{n}\left(k_{2 \perp} a\right),
\end{aligned}
$$

$$
\begin{aligned}
b_{3}= & \epsilon_{n}(i)^{n} a k_{2 \perp}^{2} J_{n}\left(k_{2 \perp} a\right), \\
b_{4}= & \epsilon_{n}(i)^{n+1} a \kappa_{z}\left(c_{11}^{(2)}-c_{13}^{(2)}\right)\left[\left(n^{2}-n-k_{2 \perp}^{2} a^{2}\right) J_{n}\left(k_{2 \perp} a\right)\right. \\
& \left.+k_{2 \perp} a J_{n+1}\left(k_{2 \perp} a\right)\right]-\left(c_{13}^{(2)}-c_{12}^{(2)}\right) \epsilon_{n}(i)^{n+1} \\
& \times a \kappa_{z}\left[n J_{n}\left(k_{2 \perp} a\right)-k_{2 \perp} a J_{n+1}\left(k_{2 \perp} a\right)\right] \\
& -\left(c_{12}^{(2)}-c_{13}^{(2)}\right) \epsilon_{n}(i)^{n+1} a n^{2} \kappa_{z} J_{n}\left(k_{2 \perp} a\right) \\
b_{5}= & \left(c_{12}^{(2)}-c_{11}^{(2)}\right) \epsilon_{n}(i)^{n+1}\left[J_{n}\left(k_{2 \perp} a\right) a \kappa_{z} n(n-1)\right. \\
& \left.-n \kappa_{z} k_{2 \perp} a^{2} J_{n+1}\left(k_{2 \perp} a\right)\right] \\
b_{6}= & c_{44}^{(2)}\left(k_{2 \perp}^{2}-\kappa_{z}^{2}\right) \epsilon_{n}(i)^{n}\left[a n J_{n}\left(k_{2 \perp} a\right)\right. \\
& \left.-k_{2 \perp} a^{2} J_{n+1}\left(k_{2 \perp} a\right)\right] .
\end{aligned}
$$

${ }^{1}$ J. J. Faran, Jr., J. Acoust. Soc. Am. 23, 405 (1951).

${ }^{2}$ R. M. White, J. Acoust. Soc. Am. 30, 771 (1958).

${ }^{3}$ L. Flax and W. G. Neubauer, J. Acoust. Soc. Am. 64, 675 (1978).

${ }^{4}$ A. Nagl, H. Uberall, P. P. Delsanto, J. D. Almar, and E. Rosario, Wave Motion 5, 235 (1983)

${ }^{5}$ N. D. Veksler, Acustica 71, 111 (1990).

${ }^{6}$ J. M. Conoir, P. Rembert, O. Lenoir, and J. L. Izbicki, J. Acoust. Soc. Am. 93, 1300 (1993).

${ }^{7}$ H. Uberall, "Surface waves in acoustics," in Physical Acoustics, edited by W. P. Mason and R. N. Thurston (Academic, New York, 1973), Vol. 10, Chap. 1.

${ }^{8}$ Y. H. Pao and C. C. Mow, Diffraction of Elastic Waves and Dynamic Stress Concentrations (Crane, Russack, New York, 1973).

${ }^{9}$ G. C. Gaunaurd, Appl. Mech. Rev. 42, 143 (1989).

${ }^{10} \mathrm{H}$. Uberall, Acoustic Resonance Scattering (Gordon and Breach Science, Philadelphia, 1992).

${ }^{11}$ N. D. Veksler, Resonance Acoustic Spectroscopy, Springer Series on Wave Phenomena (Springer-Verlag, Berlin, 1993).

${ }^{12}$ T. S. Lewis and D. W. Kraft, J. Appl. Phys. 47, 1265 (1976).

${ }^{13}$ L. Flax and H. Uberall, J. Acoust. Soc. Am. 67, 1432 (1980).

${ }^{14}$ J. P. Sessarego, J. Sageloli, R. Guillermin, and H. Uberall, J. Acoust. Soc. Am. 104, 2836 (1998).

${ }^{15}$ T. M. Hsieh, E. A. Lindgren, and M. Rosen, Ultrasonics 29, 38 (1991).

${ }^{16}$ P. Beattie, R. C. Chivers, and L. W. Anson, J. Acoust. Soc. Am. 94, No. 6, 3421 (1993).

${ }^{17}$ A. N. Sinclair and R. C. Addison, Jr., J. Acoust. Soc. Am. 94, No. 2; Pt. 1, 1126 (1993).

${ }^{18}$ A. N. Sinclair, F. Honarvar, M. R. Piggott, and M. V. Ravichandran, Proc, Intl. Conf. on Composite Materials and Energy (Technomic, Lancaster, 1995).

${ }^{19}$ W. Huang, S. Brisuda, and S. I. Rokhlin, J. Acoust. Soc. Am. 97, 807 (1995).

${ }^{20}$ W. Huang, Y. J. Wang, and S. I. Rokhlin, J. Acoust. Soc. Am. 99, 2742 (1996).

${ }^{21}$ F. Honarvar and A. N. Sinclair, Ultrasonics 36, 845 (1998).

${ }^{22}$ I. Mirsky, J. Acoust. Soc. Am. 37, 1016 (1965).

${ }^{23}$ I. Mirsky, J. Acoust. Soc. Am. 37, 1022 (1965).

${ }^{24}$ M. de Billy, J. Acoust. Soc. Am. 97, 852 (1995).

${ }^{25}$ M. de Billy, Acustica 81, 281 (1995).

${ }^{26}$ F. Honarvar and A. N. Sinclair, J. Acoust. Soc. Am. 100, 57 (1996).

${ }^{27}$ F. Honarvar, "Nondestructive evaluation of cylindrical components by resonance acoustic spectroscopy," Ph.D. thesis, University of Toronto, 1997

${ }^{28}$ S. K. Numrich, W. E. Howell, J. V. Subrahmanyam, and H. Uberall, J. Acoust. Soc. Am. 80, 1161 (1986).

${ }^{29}$ C. C. H. Guyott and P. Cawley, J. Acoust. Soc. Am. 83, 632 (1988).

${ }^{30}$ T. M. Hsieh, E. S. Lindgren, and M. Rosen, Ultrasonics 29, 38 (1991).

${ }^{31}$ S. A. Lee and D. M. Corbly, IEEE Trans. Sonics Ultrason. SU-24, 206 (1977).

${ }^{32}$ W. Maurice Ewing, W. S. Jardetzky, and F. Press, Elastic Waves in Layered Media (McGraw-Hill, New York, 1957).

${ }^{33}$ S. K. Bose and A. K. Mal, J. Met. Phys. Sol. 22, 217 (1974). 